\title{
The behaviour of exciton-polaritons
}

To the Editor - Several experimental groups have demonstrated that excitonpolaritons - quasiparticles made from a mix of light and matter - can form a coherent state (that is, a condensate in momentum space) in a semiconductor microcavity ${ }^{1-4}$. However, there is little agreement in the community regarding the nature and associated terminology of this condensate: is it a Bose-Einstein condensate (BEC), a laser, or something else? Polaritons are also sometimes described as exhibiting superfluidity. Here we wish to point out that describing polaritons and their condensate in terms of a BEC and superfluidity may be misleading.

Starting from a conventional quantum well in which no coherent excitonic state is observed, various forms of coherent states can be realized by either reducing or increasing the coupling between excitons and photons. Reducing the coupling leads to a regime in which excitons have long lifetimes, allowing them to cool down to low temperatures and thus form a coherent state whose properties are similar to a BEC. Such a regime can be realized in a system of 'indirect excitons' comprising electrons and holes in spatially separated layers. Increasing the coupling leads to the strong coupling regime, in which excitonpolaritons can form a coherent state. The limiting case of zero exciton component provides a purely photonic condensate, which is realized in conventional lasers.

The most essential properties of polariton condensates, such as long-range coherence, are governed by their photonic component ${ }^{5}$. The photonic fraction of a polariton condensate must be high enough for the coherence to form, which makes polariton condensates similar to lasers. The term 'polariton laser' therefore seems appropriate for this system.

There is, however, a clear distinction between a BEC and a polariton laser: thermal equilibrium is an essential property of $\mathrm{BEC}$, but it is not required for a laser. Experimental data show that polariton condensates can be formed without thermal equilibrium between the condensate and non-condensed polaritons ${ }^{6}$, which suggests polariton lasing rather than a BEC.

A polariton laser has both similarities and differences with a conventional photon laser. Both are characterized by spatial coherence and have a similar angular and energy distribution of emitted photons (a peak at low energies and a Boltzmann-like distribution at higher energies $\left.{ }^{7}\right)$. Both are characterized by stimulated occupation; a photon mode in the case of the photon laser or a photonexciton (polariton) mode in the case of the polariton laser. The main difference between the two is that a conventional semiconductor laser operates by exploiting a stimulated optical transition in the population-inverted electron-hole plasma, whereas the stimulated scattering of polaritons in a polariton laser does not require the electronic population to be inverted.

Another related issue is that of polariton superfluidity. As a system of interaction bosons, polaritons may in principle be subject to a phase transition towards superfluidity. The suppression of polariton scattering on a disorder potential in a structure has been interpreted in terms of polariton superfluidity ${ }^{8}$. These experiments were realized in the resonant pumping regime, where coherence was introduced to the polariton system by a pumping laser. However, scattering suppression can also be achieved by exploiting non-superfluidic effects. For example, screening a disorder potential by repulsively interacting excitons ${ }^{9}$ and parametric instabilities in a polariton system ${ }^{10}$ can suppress the scattering by more than an order of magnitude. Furthermore, experimental data indicate that the momentum scattering time remains in the picosecond range, even in the 'superfluid' regime ${ }^{8}$.

The scattering times of normal metals, in comparison, are often orders of magnitude longer. The short scattering times of the polariton system make the polariton transport regime different from the conventional superfluid regime. In addition, the polariton propagation speed is much higher than the speed of phonons in a crystal lattice ${ }^{8}$. Polaritons can therefore scatter on phonons, causing dissipation. Even if the scattering time is larger than the experiment time or polariton lifetime, such dissipation is inconsistent with superfluidity. In analogy, although the electron scattering time in a metal can be larger than the experiment time, this does not make the metal a superconductor.

Polaritons represent an interesting out-of-equilibrium bosonic system that is capable of demonstrating remarkable effects. Describing this system in terms of a BEC and superfluidity is confusing. The polariton condensate is a new type of condensate that is different from a conventional BEC. Furthermore, polariton transport is not superfluidic, although it can exhibit reduced scattering and a large coherence length. Despite the short scattering times of a polariton system, high polariton velocities may lead to large mean free paths, allowing the study of coherent polariton transport on scales accessible for optical imaging. The term 'polariton laser' does seem to be appropriate for a polariton condensate. The physics of a polariton condensate, although different from that of a BEC and superfluidity, is nevertheless rich and interesting.

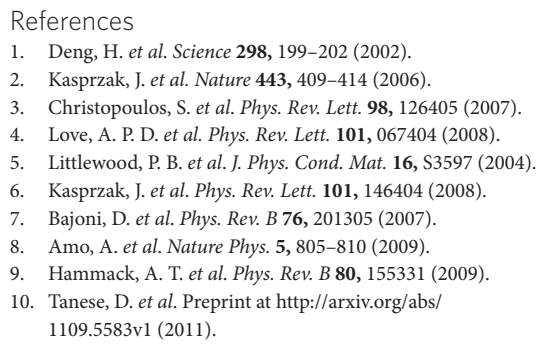

L. V. Butov ${ }^{1 \star}$ and A. V. Kavokin ${ }^{2,3}$

University of California at San Diego, 9500 Gilman Drive, La Jolla, California 920930319, USA. Université Montpellier 2, Place Eugène Bataillon-CC074, F-34095 Montpellier, France. ${ }^{3}$ University of Southampton, Highfield, Southampton SO17 1BJ, UK.

*e-mail: Ivbutov@physics.ucsd.edu 